\title{
Efectos a medio plazo de tratamientos de rehabilitación post-incendio en propiedades microbiológicas del suelo
}

\author{
Fontúrbel, M.T. ${ }^{*}$, Fernández, C. ${ }^{1}$, Vega, J.A. ${ }^{1}$ \\ ${ }^{1}$ Centro de Investigación Forestal. Lourizán. Xunta de Galicia. Aptdo. 127. 36080. Pontevedra \\ *Autor para correspondencia e-mail: maria.teresa.fonturbel.1literas@xunta.es
}

\section{Resumen}

Se evaluaron los efectos a medio plazo de varios tratamientos de estabilización del suelo, utilizados actualmente en Galicia para la rehabilitación del suelo de áreas forestales afectadas por incendios, en propiedades químicas y microbiológicas del suelo indicadoras de su calidad. El estudio se realizó en tres comunidades vegetales (carqueixal mixto, pinar/tojal, brezal mixto) que sufrieron incendios de diferente severidad, en donde se aplicaron tratamientos de mulching de paja, mulching de paja + siembra, fajinadas de troncos y mulching de corteza desfibrada, comparándose en cada caso con un control quemado sin tratamiento. Los cambios producidos por los tratamientos en las propiedades químicas y microbiológicas del suelo mineral superficial (0$2 \mathrm{~cm}$ ) fueron, en general, poco relevantes y no superaron a los asociados a las características iniciales del sitio en conjunción con el efecto del incendio. Los suelos del incendio de más alta severidad del fuego tuvieron los valores más bajos de biomasa microbiana, tasa $\mathrm{Cmic} / \mathrm{C}$ y actividad enzimática de la fosfatasa ácida en comparación a los de menor severidad. El mulching de paja favoreció una mayor diversidad funcional microbiana en cuanto a la utilización de sustratos de carbono, durante los 9 primeros meses después del incendio más severo. Los resultados indican que los suelos donde se aplicaron tratamientos de rehabilitación post-incendio no mostraron diferencias apreciables en las propiedades analizadas, y solamente se observó una ligera ventaja del tratamiento de mulching en suelos de baja calidad, por lo que podría ser el tratamiento de elección en suelos pobres y con un elevado nivel de severidad del fuego en el suelo.

Palabras clave: biomasa microbiana, diversidad funcional microbiana, fosfatasa ácida, incendios forestales, propiedades del suelo. 


\section{Introducción}

Los tratamientos de rehabilitación urgente post-fuego suelen acometerse después de grandes incendios con el objetivo de limitar la escorrentía y erosión del suelo y mitigar así los daños producidos por el fuego (Fernández et al., 2011, 2012; Robichaud et al., 2013; Vega et al., 2013 a). Son una herramienta de gran utilidad, particularmente en ecosistemas forestales como los del NO de España, una de las zonas de Europa con mayor número de incendios (San Miguel and Camia, 2009), hecho que se ve agravado por la elevada erodibilidad de los suelos quemados, la abundancia de pendientes pronunciadas y las altas precipitaciones en otoño (Vega et al., 2013 a). Debido a que los tratamientos de rehabilitación tienen un elevado coste, es necesario testar rigurosamente su efectividad, analizando su eficacia y efectos en el suelo, para poder priorizar las áreas a rehabilitar y seleccionar los tratamientos más adecuados.

Investigaciones realizadas en España han mostrado la eficacia del uso del mulching de paja o de la siembra de vegetación herbácea (Bautista et al., 1996; Badía and Martí, 2000; Pinaya et al., 2000; Fernández et al., 2011, 2012; Díaz-Raviña et al., 2012; Vega et al., 2014) desde el punto de vista de la reducción de la erosión post-incendio. Sin embargo, es muy limitada la información a nivel mundial sobre su efecto a corto y medio plazo en propiedades del suelo indicadoras de su calidad. Bautista et al. (1996) evaluaron los efectos de la aplicación de mulching de paja en suelos quemados y encontraron que uno y dos años después del tratamiento, las parcelas con mulching presentaban mayor capacidad de infiltración, contenido de humedad y tamaño medio de los agregados del suelo y una reducción de la compactación del suelo superficial respecto a parcelas quemadas sin tratamiento, e indicaron que esos cambios pueden afectar a la recuperación de la cobertura vegetal y actividad biológica del suelo, influyendo a su vez en la escorrentía y erosión del suelo. En un estudio posterior, Bautista et al. (2009) encontraron un incremento de la actividad microbiana después del mulching de paja. Sin embargo, en otros estudios no se detectaron diferencias apreciables en distintos parámetros edáficos indicadores de la calidad del suelo hasta un año después de la aplicación de mulch en relación a la siembra o la ausencia de tratamiento (Martín et al., 2010; Díaz-Raviña et al., 2012; Fontúrbel et al.., 2012; Gómez-Rey et al., 2013 a, 2013 b; Barreiro et al., 2015, Lombao et al., 2015). Huang et al. (2008) documentaron aumentos en la diversidad funcional microbiana tras la adición de restos vegetales en forma de mulch en plantaciones forestales de Australia que fueron atribuidos a cambios en las condiciones microambientales del suelo y a la cantidad y composición del carbono orgánico del suelo. Sin embargo, otros autores solo encuentran una limitada respuesta de la biomasa microbiana a la incorporación de residuos forestales al suelo para favorecer el secuestro de $\mathrm{C}$ en el ecosistema (Busse et al., 2009). Las diferentes respuestas señaladas pueden ser debidas a las características iniciales del sitio y a las condiciones meteorológicas posteriores, así como a la severidad del incendio, en el caso de tratamientos aplicados en suelos afectados por el fuego.

El objetivo de este estudio fue analizar comparativamente los efectos a medio 
plazo (3 años) de varios tratamientos de rehabilitación del suelo post-incendio (mulching de paja, mulching de paja + siembra, fajinadas de troncos y mulching de corteza desfibrada) aplicados en tres comunidades vegetales diferentes sobre propiedades bioquímicas y microbiológicas del suelo como indicadoras de su calidad.

\section{Material y métodos}

El estudio se realizó en tres dispositivos experimentales instalados en áreas forestales afectadas por los incendios de Piñor, Camba y Boiro, ocurridos en 2009 y 2010, respectivamente (Tab. 1a). Inmediatamente después del incendio, y antes de cualquier precipitación apreciable, se instalaron una serie de parcelas delimitadas con bandas de geotextiles para la recolección periódica de los sedimentos producidos en aquellas. Antes de la ejecución de los tratamientos, se efectuaron mediciones de parámetros indicadores de los niveles de severidad del fuego en el suelo, usando la clasificación en cinco niveles de severidad (muy bajo, bajo, moderado, alto y muy alto) descrita en detalle en Vega et al. (2013 a), basada en cambios visuales en la cubierta orgánica y suelo mineral superficial, que ha mostrado su utilidad para reflejar cambios en propiedades edáficas relevantes (Vega et al., 2013 b). En Camba se seleccionaron al comienzo del estudio parcelas control sin quemar, de características aparentemente similares a las que sufrieron el incendio, en las que se recogieron muestras para su posterior análisis. En Piñor, debido a la ausencia de suelos sin quemar próximos a la zona del incendio, se analizaron muestras seleccionadas aleatoriamente dentro del área del incendio caracterizadas por su aspecto visual como de severidad muy baja (Vega et al., 2013 a) que, en general, no se diferencian apreciablemente de los suelos sin quemar en sus propiedades químicas y microbiológicas (Vega et al., 2013 b). Estas muestras se recogieron inmediatamente antes de la aplicación de los tratamientos. En Boiro, no se dispuso de suelos sin quemar o prácticamente inalterados.

En los tres dispositivos experimentales, los tratamientos fueron ejecutados antes de ninguna precipitación apreciable después del incendio. En cada sitio se consideraron tres tratamientos (Tab. 1b), siendo uno de ellos un control (suelo quemado sin tratar). En Piñor, se aplicaron los tratamientos de siembra y mulching de paja de trigo, ambos realizados manualmente y procurando la distribución uniforme del material por toda la superficie de la parcela. En Camba, la paja se dispersó por el área quemada desde un helicóptero (heli-mulching) y la corteza fue distribuida manualmente. En Boiro, el mulching de paja y las fajinadas de troncos se aplicaron de forma manual. En cada dispositivo se instalaron entre 3 y 6 parcelas por tratamiento. Características más detalladas de los sitios y de los tratamientos pueden verse en Fernández y Vega $(2013,2015)$ y Vega et al. (2015).

En cada sitio, se realizó un seguimiento de los suelos quemados desde inmediatamente después de la aplicación de los tratamientos hasta 3 años después de los mismos. Se recogieron muestras de suelo mineral superficial $(0-2 \mathrm{~cm})$ en 20 puntos se- 
Tabla 1a. Características generales de los sitios de estudio

\begin{tabular}{|l|c|c|c|c|c|c|}
\hline Sitio & Provincia & $\begin{array}{c}\text { Período de } \\
\text { seguimiento }\end{array}$ & Vegetación & Clima & $\begin{array}{c}\text { Ta media } \\
\text { anual ( }\end{array}$ & $\begin{array}{c}\text { Precipitación } \\
\text { media anual } \\
(\mathrm{mm})\end{array}$ \\
\hline Piñor & Ourense & $2009-2012$ & $\begin{array}{c}\text { Carqueixal } \\
\text { mixto }\end{array}$ & Oceánico-Mediterráneo & 12 & 1241 \\
\hline Boiro & Coruña & $2010-2013$ & Pinar/tojal & Oceánico & 12 & 2025 \\
\hline Camba & Ourense & $2010-2013$ & Brezal mixto & Mediterráneo- Continental & 9 & 1100 \\
\hline
\end{tabular}

Tabla 1b. Características de los dispositivos experimentales

\begin{tabular}{|c|c|c|c|c|c|c|c|}
\hline Sitio & Sustrato & Textura & $\begin{array}{c}\text { Pendiente } \\
(\%)\end{array}$ & $\begin{array}{l}\text { Severidad } \\
\text { del fuego } \\
\text { suelo }\end{array}$ & $\begin{array}{l}\text { Tratamientos } \\
\text { ensayados }\end{array}$ & $\begin{array}{l}\text { Dosis } \\
\text { tratamiento }\end{array}$ & $\begin{array}{l}\text { Número } \\
\text { de } \\
\text { parcelas }\end{array}$ \\
\hline Piñor & Esquisto & Franca-arenosa & 36 & Alta & $\begin{array}{l}\text { Siembra / } \\
\text { Siembra+ mulching } \\
\text { paja / Control }\end{array}$ & $\begin{array}{l}\text { Mulch paja }= \\
2.5 \mathrm{Mg} \mathrm{ha}^{-1} / \\
\text { Siembra }=3 \mathrm{~g} \mathrm{~m}^{-2}\end{array}$ & 16 \\
\hline Boiro & Granito & Franca-arenosa & 35 & $\begin{array}{l}\text { Alta - } \\
\text { muy alta }\end{array}$ & $\begin{array}{l}\text { Mulching paja / } \\
\text { fajinadas troncos / } \\
\text { Control }\end{array}$ & $\begin{array}{l}\text { Mulch paja= } \\
1.0 \mathrm{Mg} \mathrm{ha}^{-1}\end{array}$ & 10 \\
\hline Camba & Esquisto & Franca-arenosa & 60 & $\begin{array}{c}\text { Moderada } \\
\text { - alta }\end{array}$ & $\begin{array}{l}\text { Mulching paja / } \\
\text { Mulching corteza / } \\
\text { Control }\end{array}$ & $\begin{array}{l}\text { Mulch paja= } \\
2.0 \mathrm{Mg} \mathrm{ha}^{-1} / \\
\text { Mulch corteza= } \\
3.5 \mathrm{Mg} \mathrm{ha}^{-1}\end{array}$ & 12 \\
\hline
\end{tabular}

leccionados aleatoriamente en cada una de las parcelas. Se realizaron las siguientes determinaciones analíticas: textura (método de la pipeta de Robinson), $\mathrm{pH}$ (1: 2.5 en agua), carbono orgánico total y nitrógeno total (método por combustión seca, LECO); biomasa microbiana (Vance et al., 1987), actividad de la fosfatasa ácida (Trasar-Cepeda et al., 2003) y perfil microbiano de utilización de sustratos de C (Garland, 1996).

Para evaluar la posible influencia de los tratamientos de rehabilitación en las propiedades del suelo de cada uno de los 3 sitios considerados, se utilizó un modelo lineal general con tratamiento y fecha de muestreo como variables independientes. Previamente, se comprobó la normalidad y homocedasticidad de los residuos. En los casos que la interacción tratamiento $\mathrm{x}$ fecha fue significativa, se aplicó un modelo lineal general para cada fecha con tratamiento como variable independiente. Se utilizó la prueba de Student-Newman-Keuls de comparaciones múltiples para determinar diferencias significativas entre tratamientos $(\alpha=0.05)$. Se usaron técnicas de regresión lineal simple para explorar posibles relaciones entre los parámetros edáficos analizados y la cantidad de erosión acumulada en el período previo a la recogida de muestras. 


\section{Resultados y discusión}

En la tabla 2 se muestran las propiedades físico-químicas de los distintos tratamientos en cada uno de los dispositivos experimentales en 4 de las fechas de análisis. La textura del suelo, con un elevado porcentaje de arena en los tres sitios, no presentó diferencias significativas atribuibles a los tratamientos entre 7 y 9 meses después de su aplicación. La humedad del suelo varió principalmente en función de la fecha de muestreo y aumentó entre 7 y 9 meses después de los tratamientos de mulching + siembra $(\mathrm{M}+\mathrm{S})$ de Boiro y mulching $(\mathrm{M})$ de Camba respecto a sus respectivos controles quemados sin tratamiento $(\mathrm{Q})$. Esos aumentos fueron de escasa cuantía y sucedieron cuando el suelo tenía muy bajo contenido de agua, lo que puede atribuirse a una mejora de las condiciones microclimáticas después del mulching, debida a la cobertura que proporciona ese material aplicado sobre la superficie del suelo (Badía y Martí, 2000; Bautista et al., 2009). El pH del suelo presentó ligeras diferencias significativas en los tratamientos $\mathrm{S}$ y $\mathrm{M}+\mathrm{S}$ de Piñor respecto a $\mathrm{Q}$ en las dos primeras fechas de análisis. En los 3 sitios y en todos los tratamientos se observó una tendencia a la disminución del pH con el paso del tiempo, lo que parece indicar que después de los aumentos iniciales, atribuidos a los aportes de cationes desde las cenizas y combustión de la materia orgánica (Certini, 2005; Mataix-Solera et al., 2009), se produce una disminución debida al lavado y arrastre de los cationes por efecto de las lluvias. El descenso de $\mathrm{pH}$ fue más notable en Boiro, lo que está en concordancia con las altas precipitaciones que se produjeron en ese sitio. Los valores de C registrados en los 3 sitios de estudio son habituales en suelos gallegos y algo más elevados en Piñor, lo que puede deberse a un contenido inicial más alto de ese elemento, como indican los valores registrados en los suelos afectados por un nivel de severidad del fuego en el suelo muy bajo en ese sitio $(17.5 \pm 1.1 \%)$. Contrastando los valores de $\mathrm{C}$ obtenidos a nivel de parcela, con los correspondientes a las muestras tomadas inmediatamente después de incendio y asignadas a cinco niveles visuales de severidad, la severidad media de la parcela corresponde a un nivel entre moderado $(12.5 \pm 0.9 \%)$ y alto $(7.8 \pm 0.5 \%)$ en ese sitio (Vega et al., 2013 b). En Camba, el C de los suelos testigo sin quemar $(\mathrm{C}=15.9 \pm 1.1 \%)$ fue también notablemente más alto que el de los que sufrieron el incendio. La erosión acumulada en el período previo a la recogida de muestras mostró una estrecha asociación negativa con el contenido de C del suelo en los dispositivos de Piñor y Boiro en las tres primeras fechas de análisis. En el primero de los sitios la erosión varió entre 0.01 y $2.95 \mathrm{t} \mathrm{ha}^{-1}$ y en el segundo, entre 0.37 y 9.93 $\mathrm{t} \mathrm{ha}^{-1}$ en esas fechas de muestreo. En Piñor, los valores del coeficiente de correlación lineal de Pearson variaron entre -0.63 y -0.71 ( $\mathrm{p}=0.011$ y $\mathrm{p}=0.003$, respectivamente; $n=15)$ y en Boiro, entre -0.69 y -0.73 ( $\mathrm{p}=0.014$ y $\mathrm{p}=0.007$, respectivamente; $\mathrm{n}=12$ ). El contenido de carbono orgánico del suelo, del que depende la estructura y agregación del suelo, suele disminuir después de fuegos severos (Vega et al., 2013 b) y ello puede reflejarse en un aumento de la susceptibilidad del suelo a la erosión (Benito et al., 2014). 
Tabla 2. Características físico-químicas de los suelos tratados y quemados sin tratar en diferentes fechas después de la aplicación de los tratamientos.

\begin{tabular}{|c|c|c|c|c|c|c|c|c|c|c|}
\hline & & & Piñor & & & Boiro & & & Camba & \\
\hline & meses & Q & $\mathrm{S}$ & $\mathrm{M}+\mathrm{S}$ & Q & $\mathrm{M}+\mathrm{S}$ & Faj. & Q & M & Trit. \\
\hline Arena & $7-9$ & $74.6 \pm 1.1$ & $77.4 \pm 0.9$ & $77.7 \pm 1.2$ & $82.5 \pm 0.9$ & $81.2 \pm 1.9$ & $79.7 \pm 0.3$ & $79.0 \pm 1.4$ & $79.1 \pm 0.7$ & $76.8 \pm 1.1$ \\
\hline Limo & $7-9$ & $14.7 \pm 0.8$ & $13.2 \pm 0.6$ & $13.2 \pm 1.1$ & $9.3 \pm 0.5$ & $10.6 \pm 1.2$ & $10.4 \pm 0.2$ & $15.5 \pm 1.1$ & $15.3 \pm 0.4$ & $17.0 \pm 0.9$ \\
\hline Arcilla & $7-9$ & $10.7 \pm 0.9$ & $9.3 \pm 0.7$ & $9.1 \pm 0.4$ & $8.1 \pm 0.4$ & $8.3 \pm 1.1$ & $9.9 \pm 0.4$ & $5.6 \pm 0.4$ & $5.6 \pm 0.2$ & $6.1 \pm 0.3$ \\
\hline \multirow[t]{4}{*}{ Hum } & 0 & $22.6 \pm 0.9$ & $21.0 \pm 1.0$ & $21.3 \pm 0.9$ & $2.2 \pm 0.2$ & $2.8 \pm 0.1$ & $1.6 \pm 0.1$ & $20.6 \pm 0.5$ & $20.8 \pm 1.0$ & $21.4 \pm 0.5$ \\
\hline & $7-9$ & $24.4 \pm 0.8$ & $22.4 \pm 1.1$ & $24.0 \pm 1.1$ & $3.4 \pm 0.6$ & $6.5 * \pm 1.6$ & $2.5 \pm 0.1$ & $4.1 \pm 0.29$ & $9.1 * \pm 0.8$ & $6.9 \pm 1.5$ \\
\hline & $24-28$ & $27.9 \pm 0.9$ & $25.2 \pm 1.5$ & $29.5 \pm 1.7$ & $21.3 \pm 1.1$ & $23.8 \pm 1.3$ & $19.3 \pm 2.6$ & $12.9 \pm 1.0$ & $13.1 \pm 2.9$ & $12.7 \pm 1.0$ \\
\hline & 36 & $19.3 \pm 0.8$ & $19.5 \pm 1.0$ & $18.5 \pm 1.1$ & $16.8 \pm 1.4$ & $14.0 \pm 1.0$ & $18.6 \pm 2.1$ & $23.8 \pm 0.5$ & $25.2 \pm 1.2$ & $26.5 \pm 1.2$ \\
\hline \multirow[t]{4}{*}{$\mathrm{pH}$} & 0 & $5.02 \pm 0.06$ & $4.90 * \pm 0.03$ & $4.94 * \pm 0.04$ & $5.01 \pm 0.10$ & $4.98 \pm 0.14$ & $5.12 \pm 0.04$ & $4.76 \pm 0.05$ & $4.77 \pm 0.07$ & $4.73 \pm 0.02$ \\
\hline & $7-9$ & $5.03 \pm 0.02$ & $4.94 * \pm 0.02$ & $4.96 * \pm 0.02$ & $4.64 \pm 0.05$ & $4.60 \pm 0.13$ & $4.37 \pm 0.08$ & $4.82 \pm 0.03$ & $4.90 \pm 0.07$ & $4.78 \pm 0.05$ \\
\hline & $24-28$ & $5.05 \pm 0.06$ & $5.02 \pm 0.01$ & $5.01 \pm 0.04$ & $4.62 \pm 0.05$ & $4.54 \pm 0.10$ & $4.55 \pm 0.03$ & $4.47 \pm 0.05$ & $4.7 \pm 0.06$ & $4.53 \pm 0.10$ \\
\hline & 36 & $4.71 \pm 0.08$ & $4.77 \pm 0.07$ & $4.75 \pm 0.03$ & $4.53 \pm 0.04$ & $4.48 \pm 0.08$ & $4.51 \pm 0.05$ & $4.55 \pm 0.05$ & $4.55 \pm 0.04$ & $4.56 \pm 0.02$ \\
\hline \multirow[t]{4}{*}{$\% \mathrm{C}$} & 0 & $8.4 \pm 0.4$ & $8.9 \pm 0.3$ & $9.4 \pm 0.3$ & $6.7 \pm 0.3$ & $7.9 \pm 0.3$ & $6.8 \pm 0.4$ & $8.4 \pm 0.3$ & $8.6 \pm 0.5$ & $7.9 \pm 0.5$ \\
\hline & $7-9$ & $9.0 \pm 0.4$ & $9.3 \pm 0.3$ & $10.0 \pm 0.3$ & $8.2 \pm 0.6$ & $9.6 \pm 0.6$ & $8.3 \pm 0.4$ & $8.4 \pm 0.5$ & $7.4 \pm 0.4$ & $7.7 \pm 0.2$ \\
\hline & $24-28$ & $9.4 \pm 0.4$ & $9.4 \pm 0.4$ & $10.6 \pm 0.3$ & $7.2 \pm 0.3$ & $7.4 \pm 0.4$ & $7.3 \pm 0.3$ & $7.1 \pm 0.2$ & $7.1 \pm 0.3$ & $7.8 \pm 0.6$ \\
\hline & 36 & $10.2 \pm 0.6$ & $9.5 \pm 0.5$ & $10.8 \pm 0.6$ & $7.6 \pm 0.2$ & $8.3 \pm 0.6$ & $7.6 \pm 0.2$ & $6.9 \pm 0.2$ & $7.3 \pm 0.4$ & $7.6 \pm 0.9$ \\
\hline \multirow[t]{4}{*}{$\% \mathrm{~N}$} & 0 & $0.63 \pm 0.02$ & $0.63 \pm 0.01$ & $0.68 \pm 0.02$ & $0.60 \pm 0.02$ & $0.68 \pm 0.03$ & $0.61 \pm 0.04$ & $0.43 \pm 0.01$ & $0.43 \pm 0.02$ & $0.40 \pm 0.03$ \\
\hline & $7-9$ & $0.63 \pm 0.02$ & $0.63 \pm 0.01$ & $0.68 \pm 0.02$ & $0.58 \pm 0.04$ & $0.66 \pm 0.02$ & $0.63 \pm 0.05$ & $0.47 \pm 0.02$ & $0.42 \pm 0.02$ & $0.42 \pm 0.00$ \\
\hline & $24-28$ & $0.66 \pm 0.03$ & $0.63 \pm 0.02$ & $0.73 * \pm 0.02$ & $0.53 \pm 0.03$ & $0.56 \pm 0.03$ & $0.5 \pm 0.04$ & $0.41 \pm 0.01$ & $0.4 \pm 0.02$ & $0.43 \pm 0.03$ \\
\hline & 36 & $0.64 \pm 0.03$ & $0.61 \pm 0.03$ & $0.69 \pm 0.04$ & $0.53 \pm 0.02$ & $0.59 \pm 0.04$ & $0.5 \pm 0.02$ & $0.44 \pm 0.01$ & $0.46 \pm 0.02$ & $0.47 \pm 0.04$ \\
\hline \multirow[t]{4}{*}{$\mathrm{C} / \mathrm{N}$} & 0 & $14.3 \pm 0.33$ & $14.8 \pm 0.29$ & $14.8 \pm 0.15$ & $11.2 \pm 0.5$ & $11.6 \pm 0.3$ & $11.0 \pm 0.4$ & $19.6 \pm 0.3$ & $20.0 \pm 0.3$ & $19.5 \pm 0.1$ \\
\hline & $7-9$ & $14.3 \pm 0.33$ & $14.8 \pm 0.29$ & $14.8 \pm 0.15$ & $14.1 \pm 0.4$ & $14.5 \pm 0.5$ & $13.2 \pm 0.7$ & $19.6 \pm 0.3$ & $20.0 \pm 0.3$ & $19.5 \pm 0.1$ \\
\hline & $24-28$ & $14.2 \pm 0.47$ & $14.8 \pm 0.25$ & $14.5 \pm 0.19$ & $13.7 \pm 0.4$ & $13.4 \pm 0.2$ & $14.9 \pm 0.5$ & $17.6 \pm 0.2$ & $17.8 \pm 0.2$ & $18.1 \pm 0.2$ \\
\hline & 36 & $15.9 \pm 0.46$ & $15.5 \pm 0.21$ & $15.8 \pm 0.15$ & $14.4 \pm 0.4$ & $13.9 \pm 0.3$ & $15.2 \pm 0.4$ & $15.7 \pm 0.2$ & $15.8 \pm 0.2$ & $16.0 \pm 0.5$ \\
\hline
\end{tabular}

Arena, limo y arcilla, expresados en \%. Hum: \% de humedad del suelo en el momento de la recogida de muestras.

Símbolos tratamientos: Q: quemado, sin tratamiento; S: siembra; M: mulching de paja; Faj.: fajinadas de troncos; Trit: cortezas trituradas.

*: diferencias significativas $(\mathrm{p}<0.05)$ respecto al quemado sin tratamiento, para cada fecha y sitio.

Los parámetros microbianos relativos a la biomasa, actividad y diversidad analizados (Fig. 1 a 3) presentaron mayor variabilidad que los químicos, aunque, en general, las diferencias entre tratamientos también fueron escasas. Los suelos de Boiro tuvieron, a lo largo de los 3 años de estudio, los valores más bajos de $\mathrm{C}$ de la bioma- 
a)

Piñor

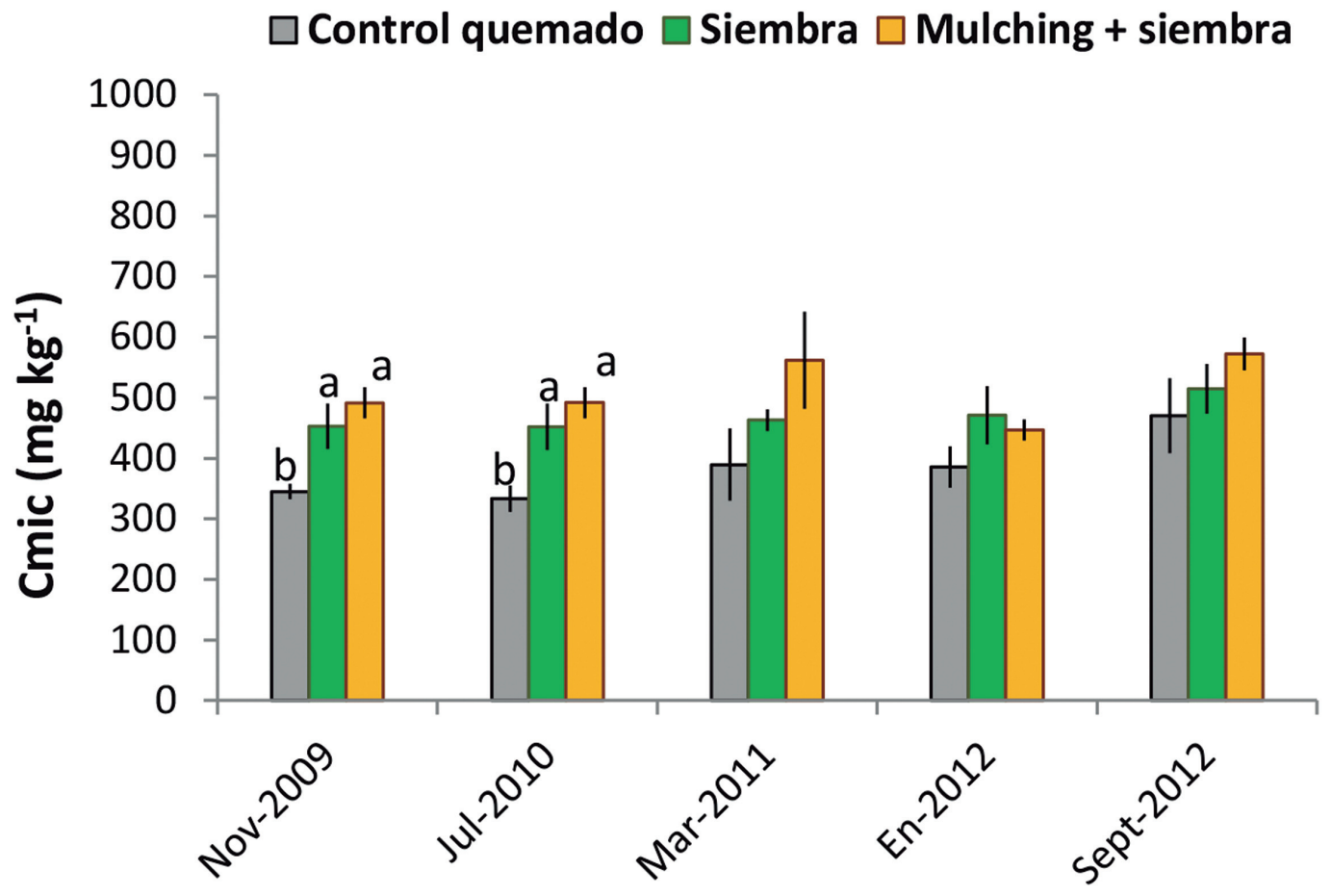

b)

Piñor

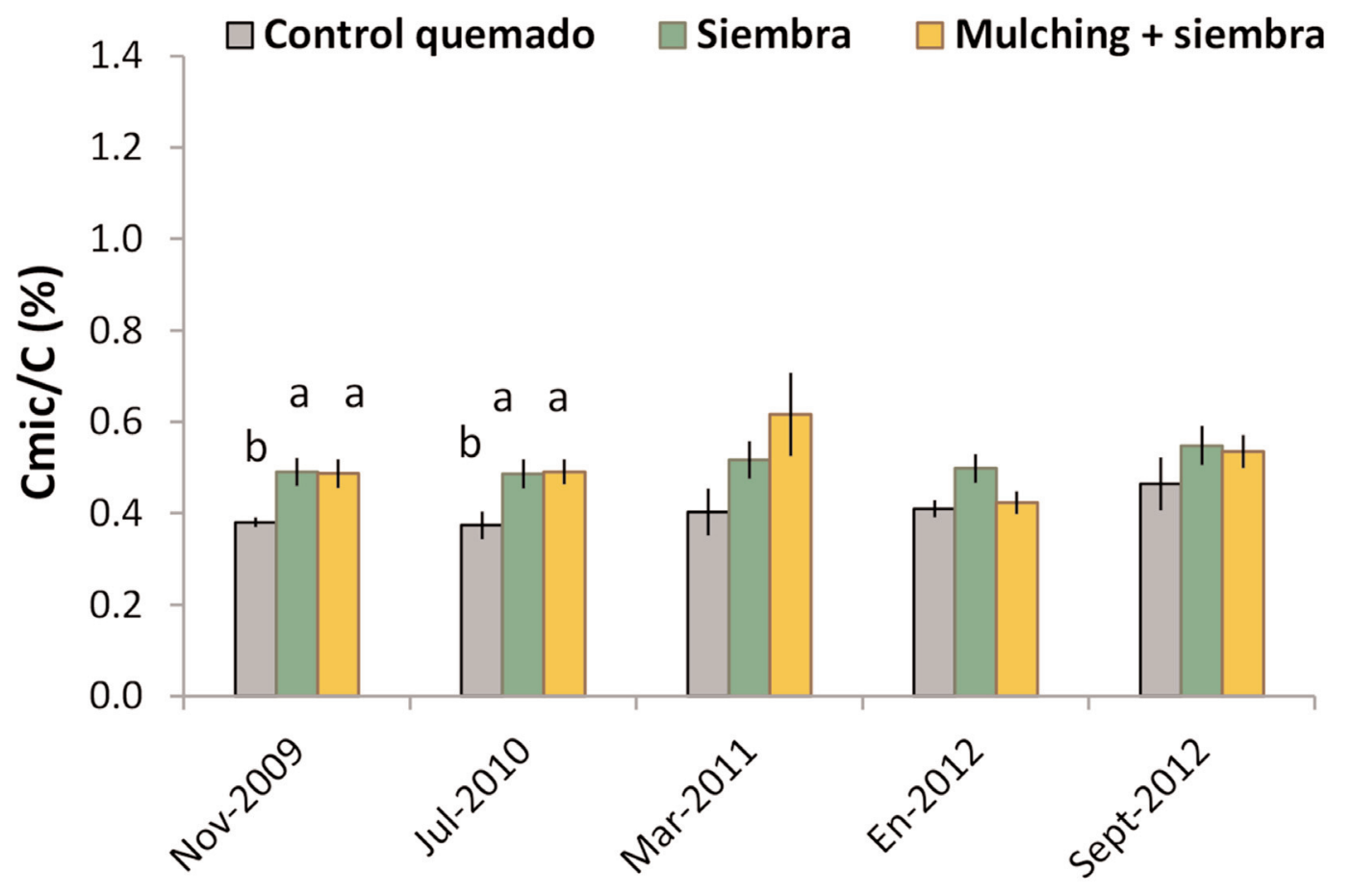


a)

\title{
Boiro
}

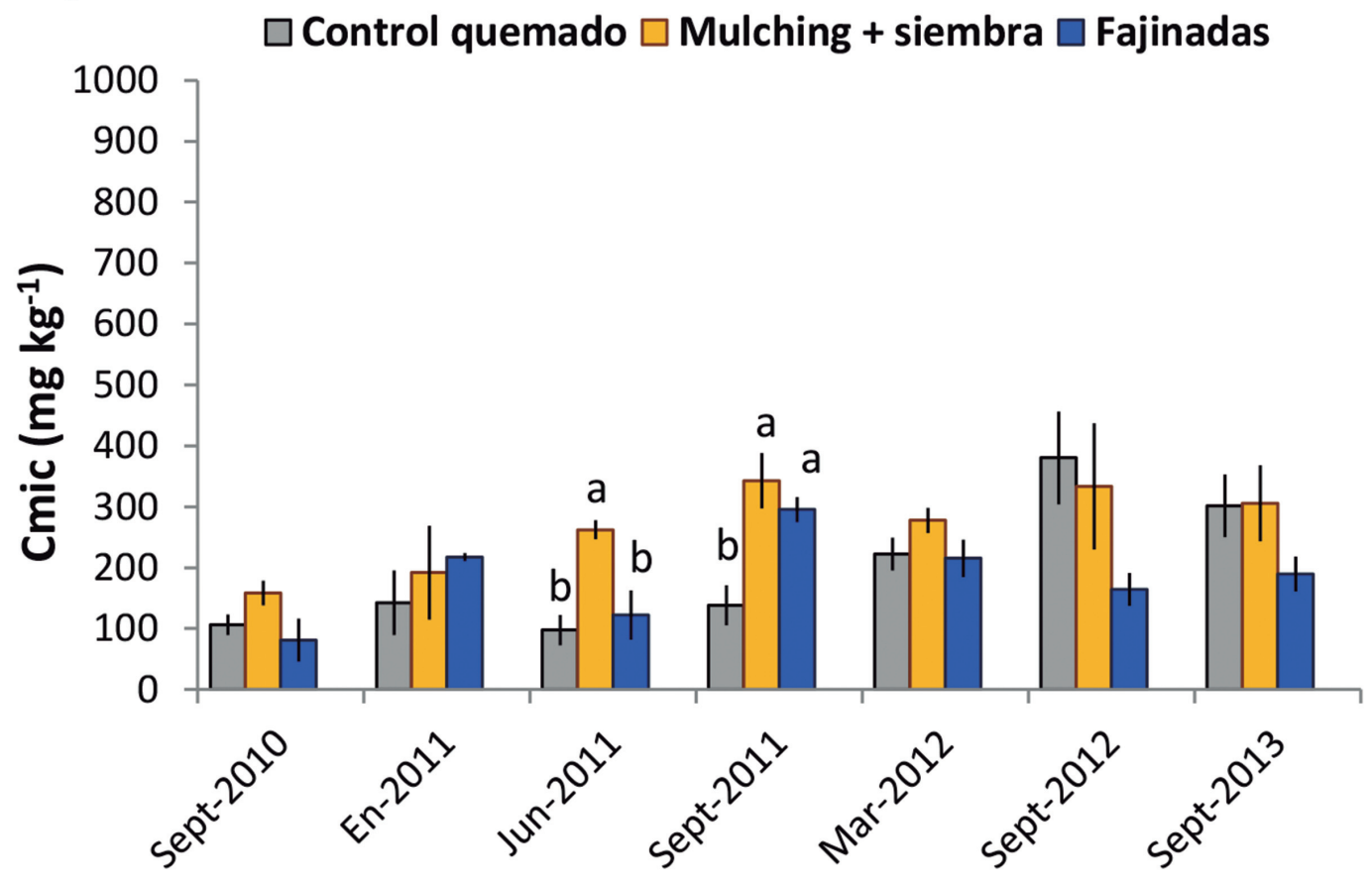

b)

\author{
Boiro
}

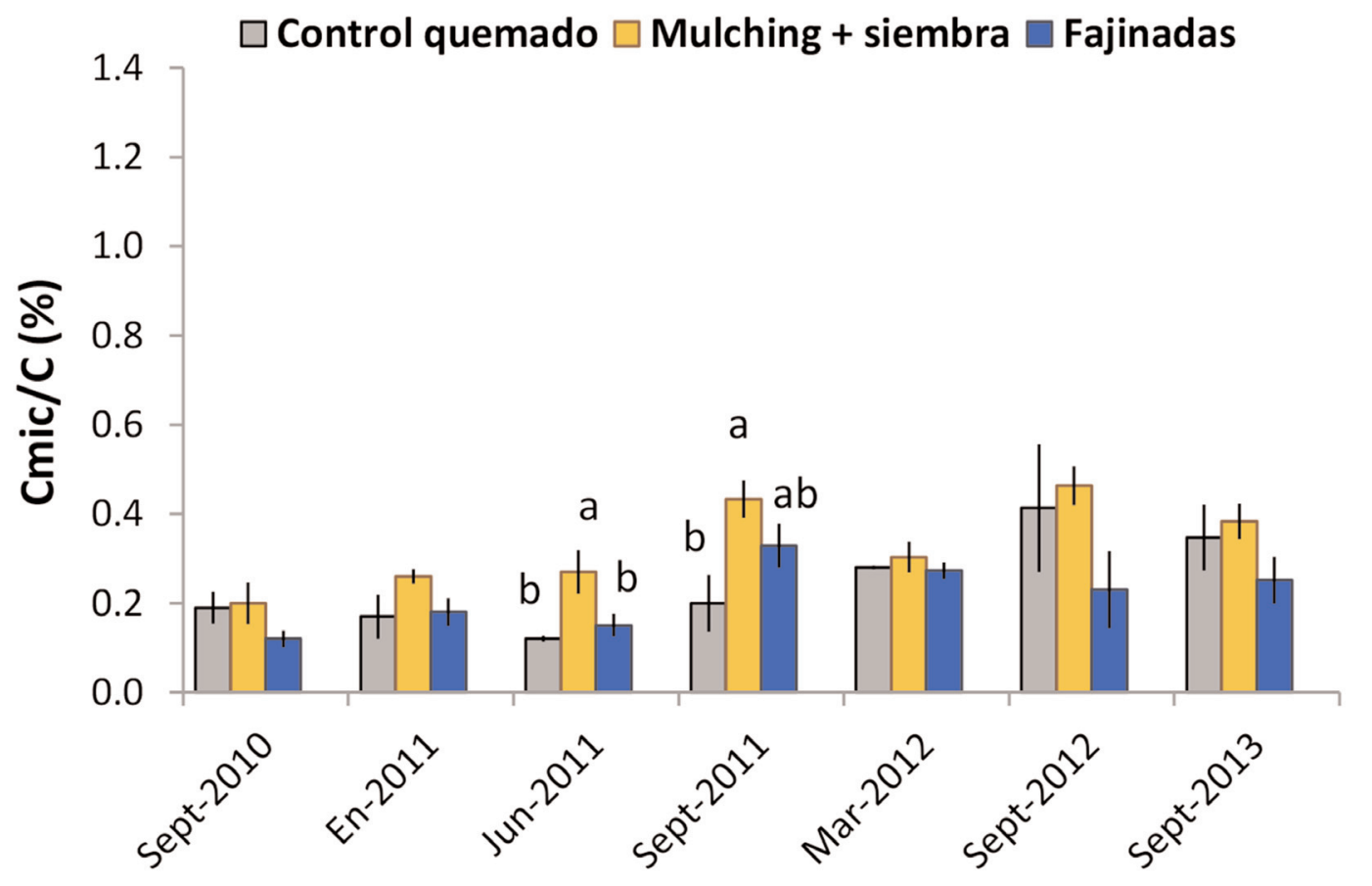


a)

Camba

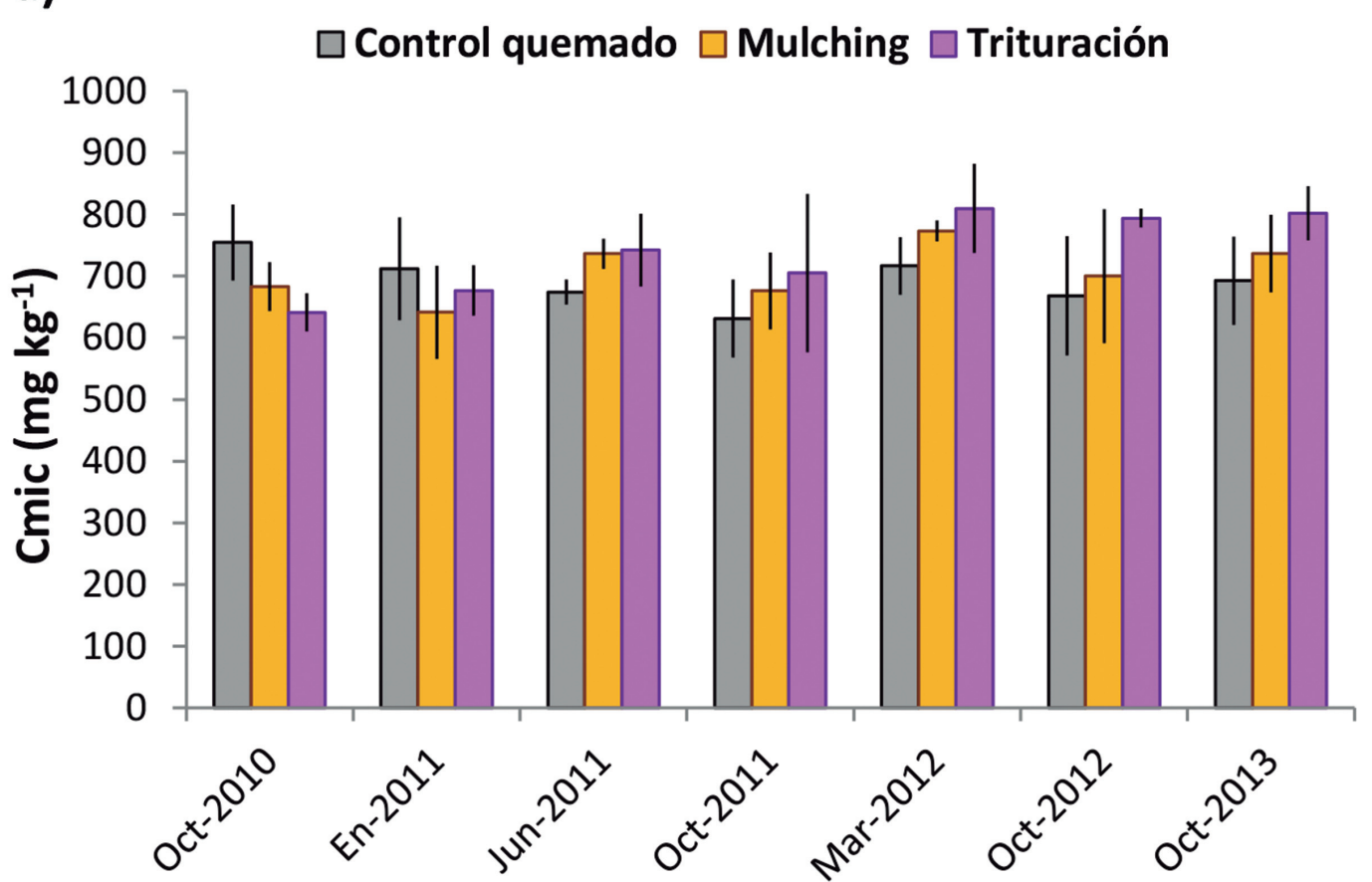

b)

\section{Camba}

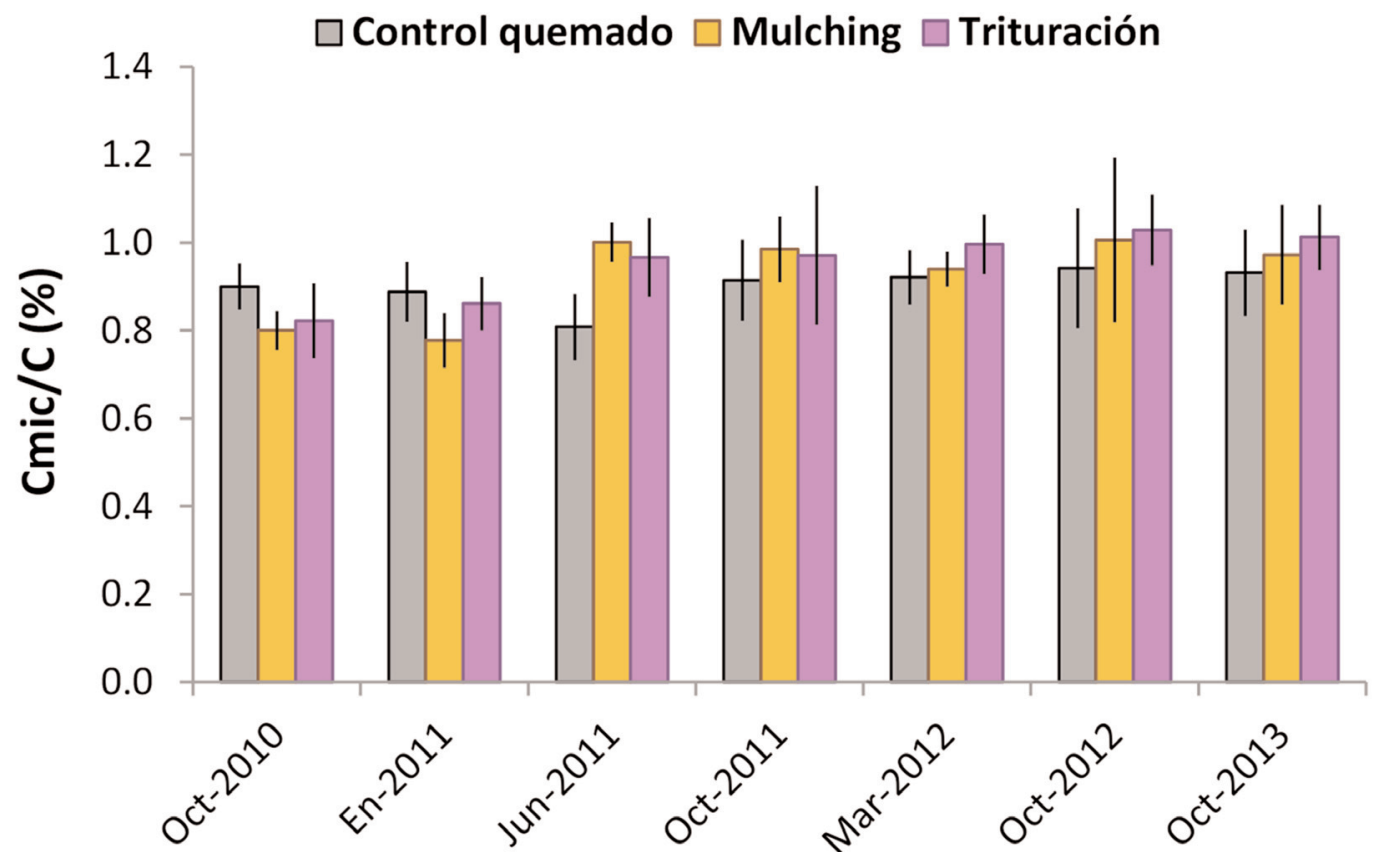

Figura 1. Efectos de los tratamientos de rehabilitación post-fuego aplicados en los dispositivos experimentales de Piñor, Boiro y Camba en: a) $\mathrm{C}$ de la biomasa microbiana (Cmic) y b) tasa $\mathrm{Cmic} / \mathrm{C}$ del suelo mineral superficial $(0-2 \mathrm{~cm})$ a lo largo de 3 años de estudio. Letras distintas en cada fecha indican diferencias significativas $(\mathrm{p}<0.05)$ según el test de Student-Newman-Keuls. 
sa microbiana (Cmic) (Fig. 1 a), mientras que en Camba los valores de Cmic fueron los más favorables, presentando Piñor valores intermedios, lo que parece reflejar las diferencias en la severidad del incendio. En los suelos quemados de Piñor categorizados como de severidad alta y moderada (Vega et al., 2013 b), analizados inmediatamente después del incendio, se obtuvieron valores de Cmic significativamente más bajos ( $118 \pm 32$ y $305 \pm 53 \mathrm{mg} \mathrm{kg}^{-1}$, respectivamente) que en los quemados con severidad muy baja ( $\left.956 \pm 91 \mathrm{mg} \mathrm{kg}^{-1}\right)$. Esto indica que los valores de Cmic, a nivel de parcela, fueron algo más elevados que los correspondientes a los obtenidos para muestras seleccionadas por su categoría de severidad. La diferencia podría ser debida a que los valores medios de parcela pudieron "diluir" el efecto de la severidad registrado a nivel puntual. Por su parte, los suelos no quemados de Camba también registraron valores de Cmic considerablemente más altos $\left(1590 \pm 104 \mathrm{mg} \mathrm{kg}^{-1}\right)$ que los quemados, lo que permite suponer que en esos suelos se habían alterado notablemente sus propiedades a consecuencia del incendio. Díaz-Raviña et al. (2012) también encontraron una reducción significativa de Cmic, una y 14 semanas después de un incendio de alta severidad, al mismo tiempo que no observaron ningún efecto de los tratamientos de mulching y siembra respecto a la no aplicación de los mismos. Otros estudios también indican ausencia de cambios significativos en el Cmic del suelo después de tratamientos de rehabilitación post-incendio respecto a la no actuación (Fontúrbel et al., 2012; Barreiro et al., 2014, Lombao et al., 2015). La tasa Cmic/C (Fig. 1 b) mostró una pauta similar a la de Cmic, con valores más bajos en Boiro y más altos en Camba. Una fuerte reducción de la tasa $\mathrm{Cmic} / \mathrm{C}$ ha sido detectada en suelos gallegos severamente afectados por incendios (Vega et al., $2013 \mathrm{~b}$ ), lo que puede ser debido a la reducción de las fracciones lábiles de $\mathrm{C}$ y a la condensación de compuestos de bajo peso molecular en macromoléculas resistentes a la degradación microbiana (González-Pérez et al., 2004). Los incrementos de Cmic/C en las parcelas tratadas de Piñor (entre la aplicación y los ocho meses de los tratamientos) y Boiro (entre los 9 y 12 meses), reflejan una mayor proporción del C lábil del suelo, y un uso más eficiente de los sustratos orgánicos por la biomasa microbiana. La erosión acumulada mostró una correlación negativa significativa con Cmic en los dispositivos de Piñor y Boiro en las dos primeras fechas de análisis. En Piñor, los valores del coeficiente de correlación lineal de Pearson fueron -0.62 y $-0.69(\mathrm{p}=0.013$ y $\mathrm{p}=0.005)$ para la primera y segunda fecha de muestro, respectivamente y en Boiro fueron -0.63 y -0.66 $(\mathrm{p}=0.029$ y $\mathrm{p}=0.020$, respectivamente).

La actividad de la enzima fosfatasa ácida (Fig. 2) fue bastante más reducida en Boiro que en los otros dos sitios y apenas se detectaron diferencias significativas entre tratamientos. Dada la sensibilidad de las actividades enzimáticas a las condiciones microambientales, cabía esperar que la cubierta protectora ofrecida por el mulching de paja pudiera haber afectado positivamente a los suelos tratados; sin embargo, ese efecto no fue detectado, lo que sugiere que o esos cambios no tuvieron la magnitud suficiente o bien, que otros factores puedan haber limitado las actividades enzimáticas. Resultados previos, tampoco indican cambios significativos en diversas actividades enzimáticas el primer año después de la aplicación de tratamientos de rehabilitación tras incendios o fuegos experimentales aunque sí después de incendios 


\section{Piñor}
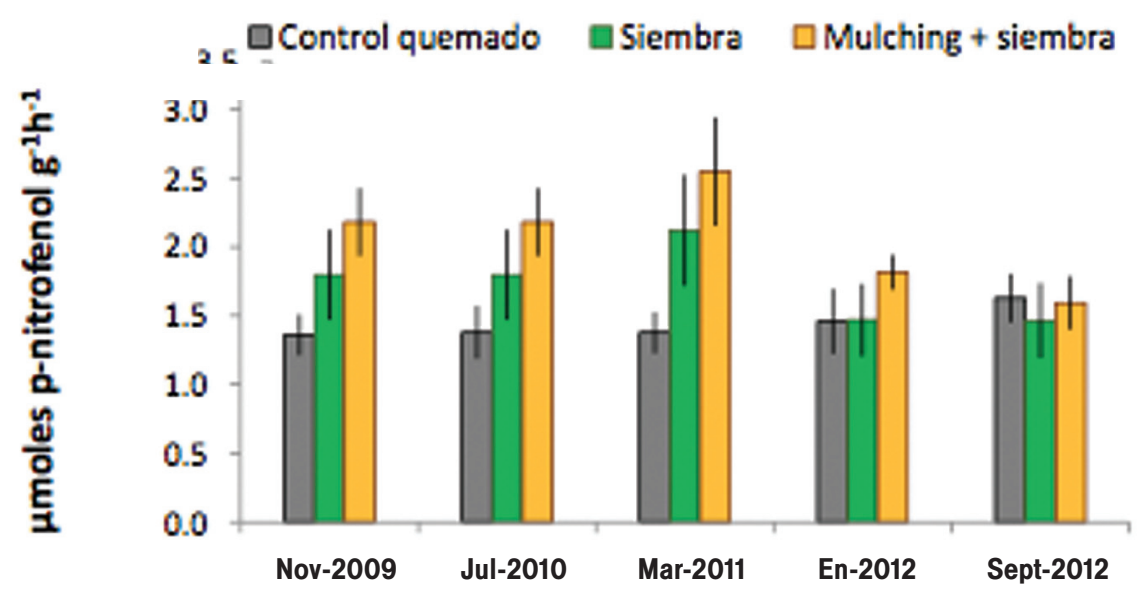

\section{Boiro}

\section{$\square$ Control quemado $\square$ Mulching + siembra $\square$ Fajinadas}

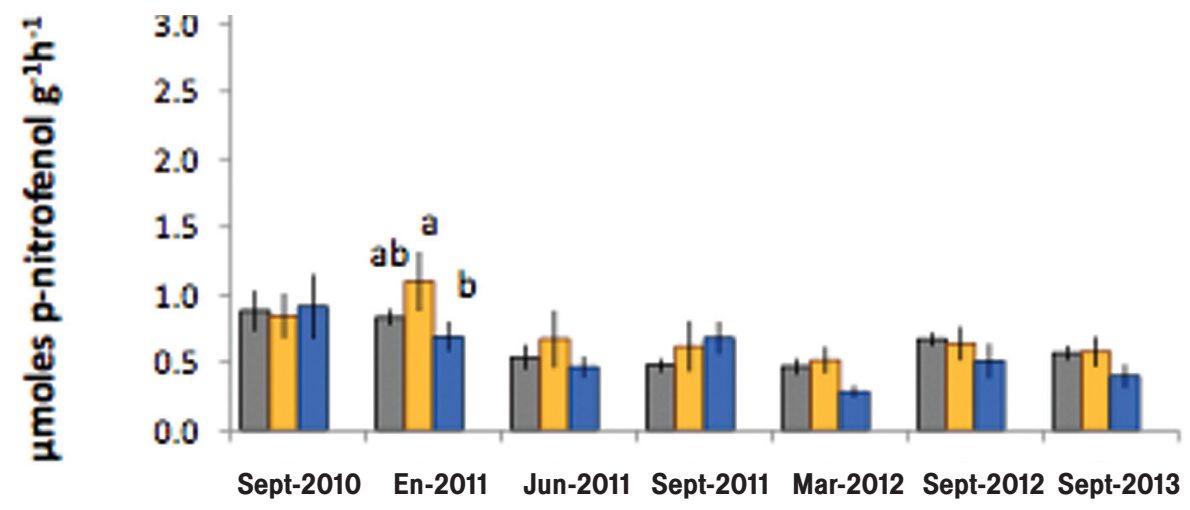

\section{Camba}

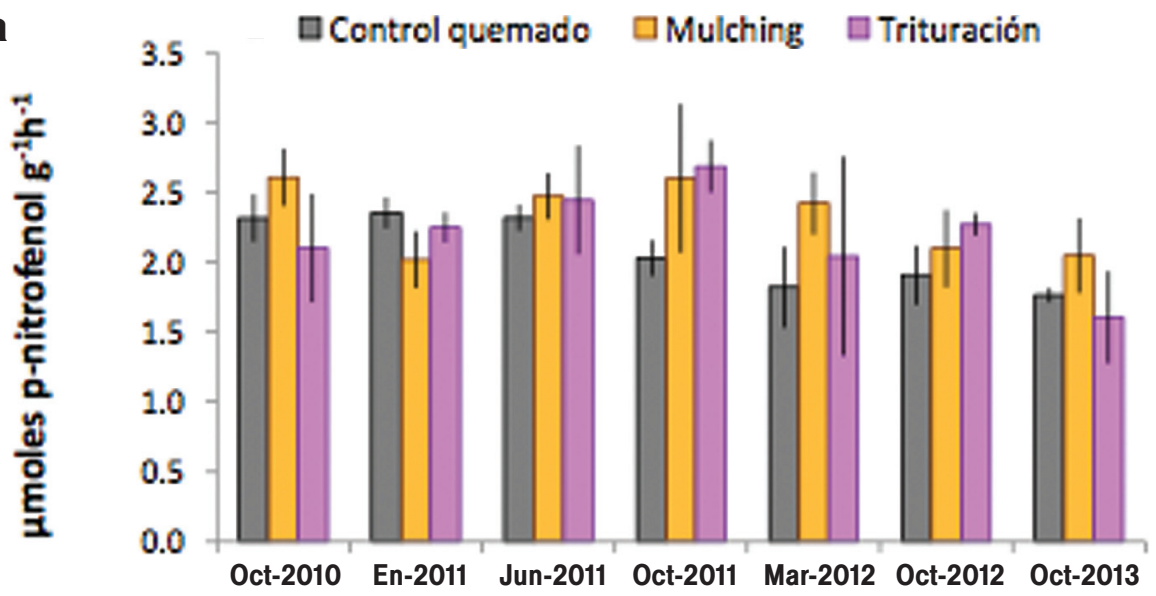

Figura 2. Efectos de los tratamientos de rehabilitación post-fuego aplicados en los dispositivos experimentales de Piñor, Boiro y Camba en la actividad de la fosfatasa (mmoles p-nitrofenol g-1 h-1 del suelo mineral superficial $(0-2 \mathrm{~cm})$ a lo largo de 3 años de estudio. Letras distintas en cada fecha indican diferencias significativas $(\mathrm{p}<0.05)$ según el test de Student-Newman-Keuls. 
o fuegos experimentales (Díaz-Raviña et al., 2012; Fontúrbel et al., 2012; Lombao et al., 2015).

Los índices de diversidad microbiana (utilización media de sustratos de C e índice de diversidad de Shannon) (Figura $3 a$, 3b) reflejaron una respuesta relativamente diferente a la de la biomasa y actividad enzimática microbiana. Así, Boiro con muy bajos valores de los anteriores parámetros, presentó, sin embargo, valores relativamente similares de diversidad funcional que los de los otros dos sitios, lo que sugiere que esos parámetros reflejan diferentes aspectos de la microbiota del suelo y coincide con resultados previos (Fontúrbel et al., 2012). El tratamiento de mulching fomentó en Boiro un ligero mayor uso potencial de sustratos de carbono, especialmente en las tres primeras fechas siguientes a la aplicación de los tratamientos, aunque debido a la alta variabilidad registrada no llegaron a detectarse diferencias significativas. Un aumento del uso microbiano de sustratos de $\mathrm{C}$ en suelos quemados respecto a suelos no quemados ha sido encontrado por diversos autores, si bien después de fuegos de severidad baja o moderada (Staddon et al., 1997; D'Ascoli et al., 2005; Fontúrbel et al., 2012; Barreiro et al., 2014), lo que ha sido atribuido a la proliferación de bacterias adaptadas al uso de distintas fuentes de $\mathrm{C}$ y al aumento de nutrientes después del fuego. Respecto al efecto de los tratamientos, los dos últimos estudios indican ausencia de diferencias achacables al mulching o a la siembra respecto a la no actuación. En contraste, en estudios realizados sin la intervención del fuego, la adición de enmiendas orgánicas en forma de mulching y la de residuos vegetales triturados ha propiciado un aumento de la diversidad funcional microbiana respecto a suelos control y se ha relacionado con la mayor disponibilidad de $\mathrm{C}$ en los suelos tratados (García, 2013; Huang et al., 2008). Los incrementos de Cmic y Cmic/C detectados en Boiro, podrían estar en relación con el aumento de $\mathrm{C}$ lábil y de la diversidad funcional en las parcelas tratadas, aunque solo se encontraron asociaciones estadísticas significativas débiles entre esas variables.

En conjunto, los resultados obtenidos muestran que los cambios producidos por los tratamientos fueron poco relevantes y no superaron a los asociados a las características iniciales del sitio en conjunción con el efecto del incendio. La respuesta registrada en Boiro, con valores más reducidos de biomasa microbiana, tasa $\mathrm{Cmic} / \mathrm{C} \mathrm{y}$ actividad enzimática de la fosfatasa, están en concordancia con el mayor nivel de severidad del fuego en el suelo, la más alta erosión y la más lenta recuperación de la vegetación en ese sitio. Además, los incrementos transitorios de los valores de los anteriores parámetros en el dispositivo y los de la diversidad funcional microbiana en las parcelas tratadas de Boiro reflejan que podría haberse producido una leve mejoría de las propiedades y condiciones que afectan a los procesos microbianos correspondientes. Todo ello parece indicar que los parámetros de calidad del suelo analizados no sufrieron cambios apreciables tras los tratamientos y solo se aprecia una ligera ventaja del tratamiento de mulching en suelos de baja calidad, por lo que podría ser el tratamiento de elección en suelos pobres y con un elevado nivel de severidad del fuego en el suelo. 


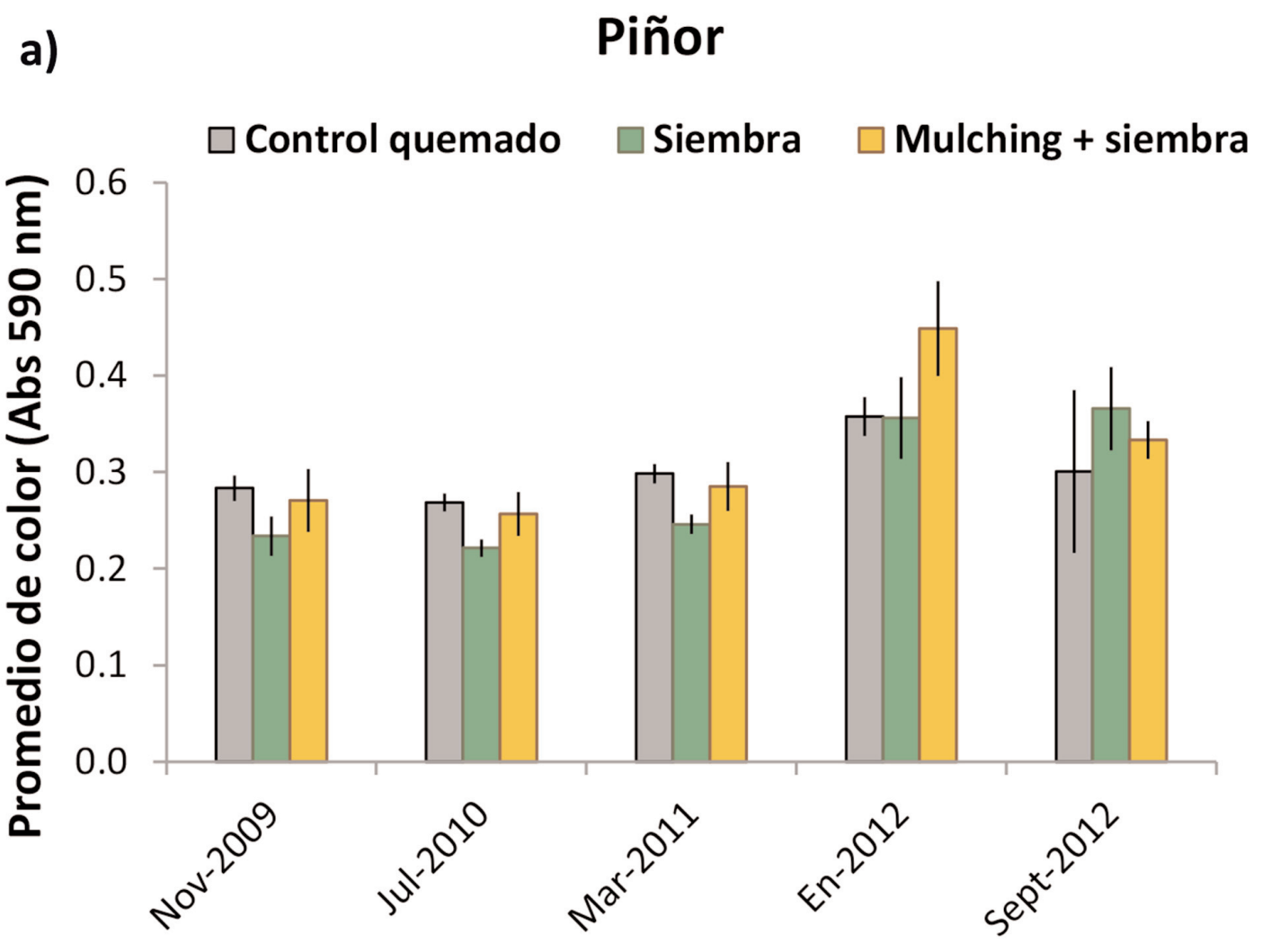

b)

Piñor

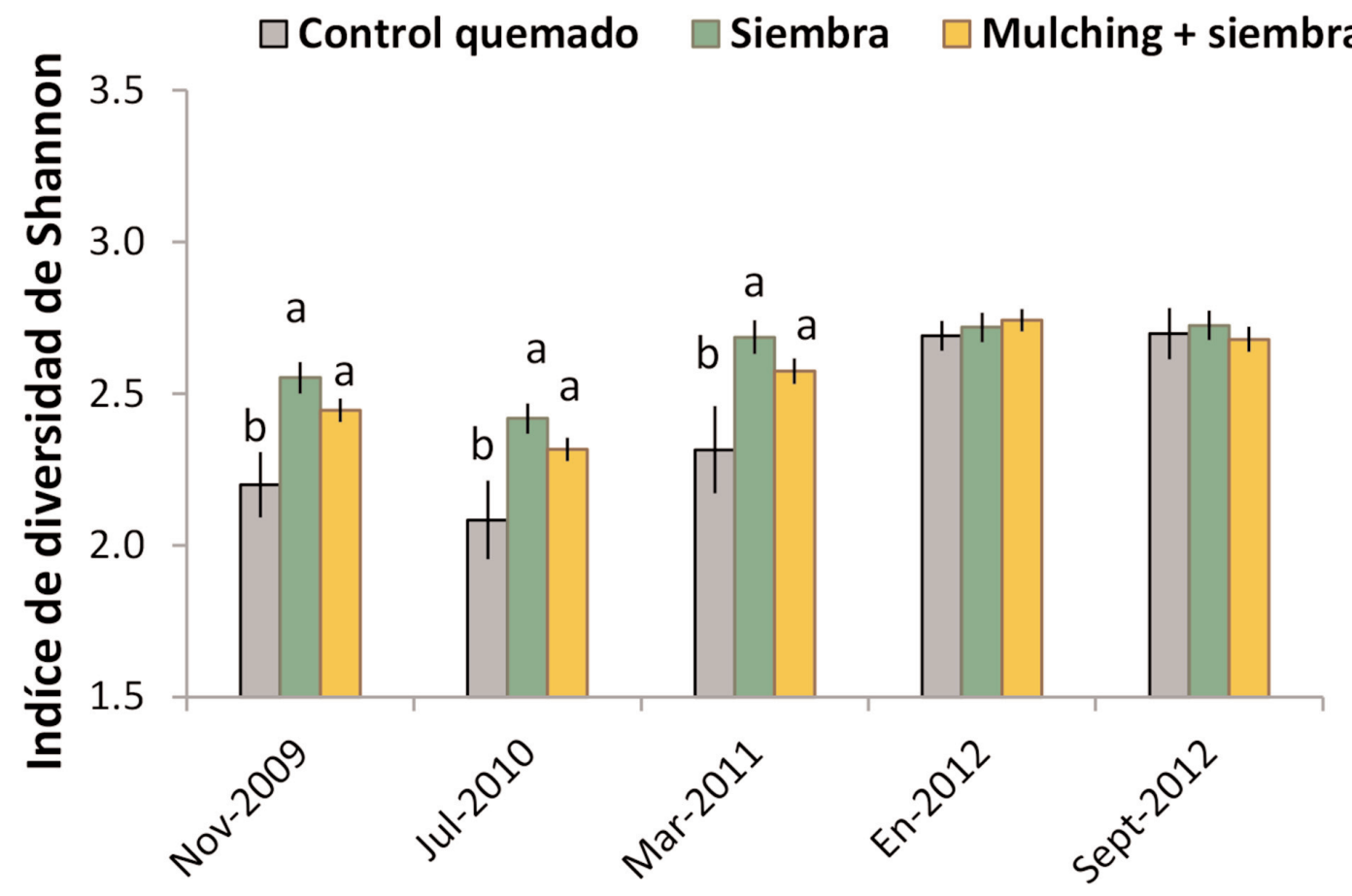


a)

Boiro

$\square$ Control quemado $1 \square$ Mulching + siembra $\square$ Fajinadas
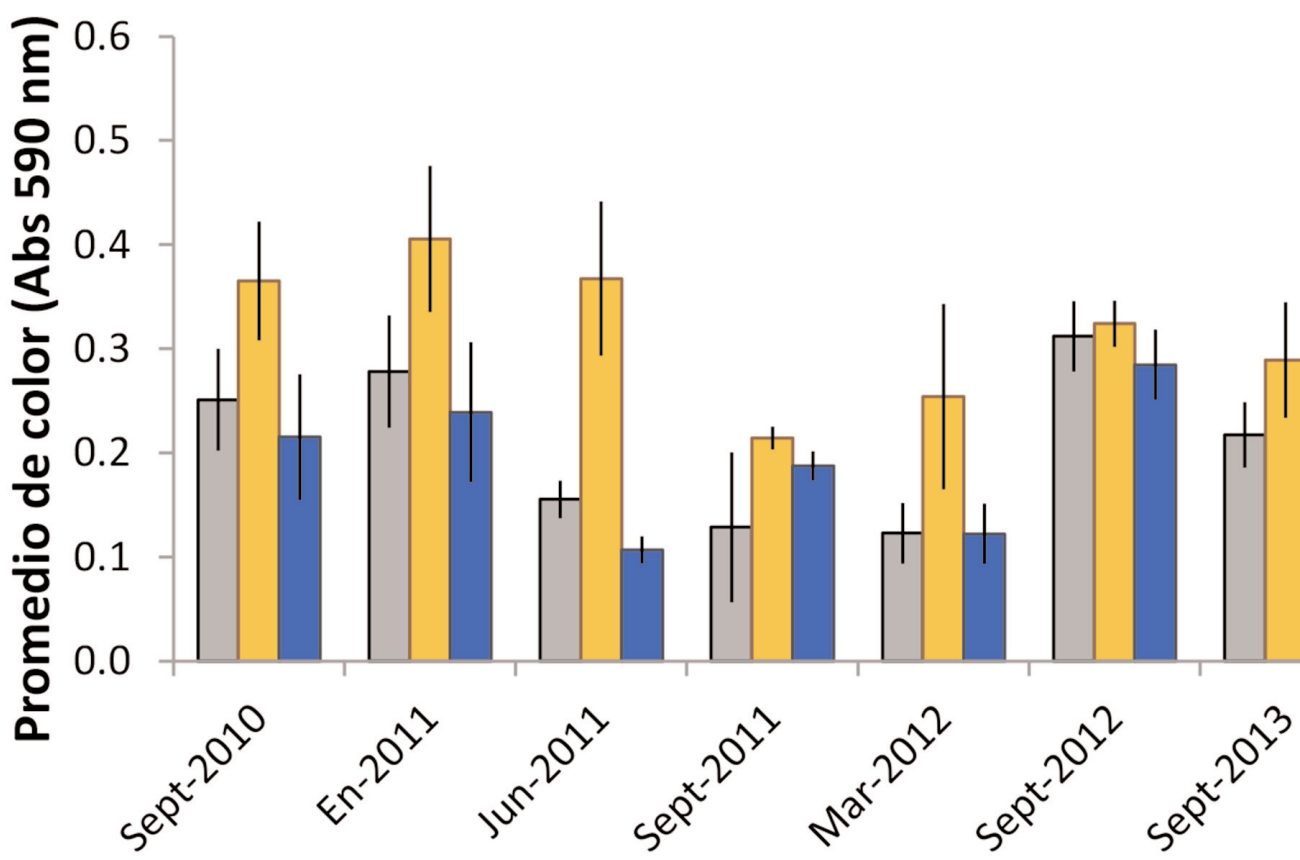

b)

Boiro

$\square$ Control quemado $1 \quad \square$ Mulching + siembra $\square$ Fajinadas

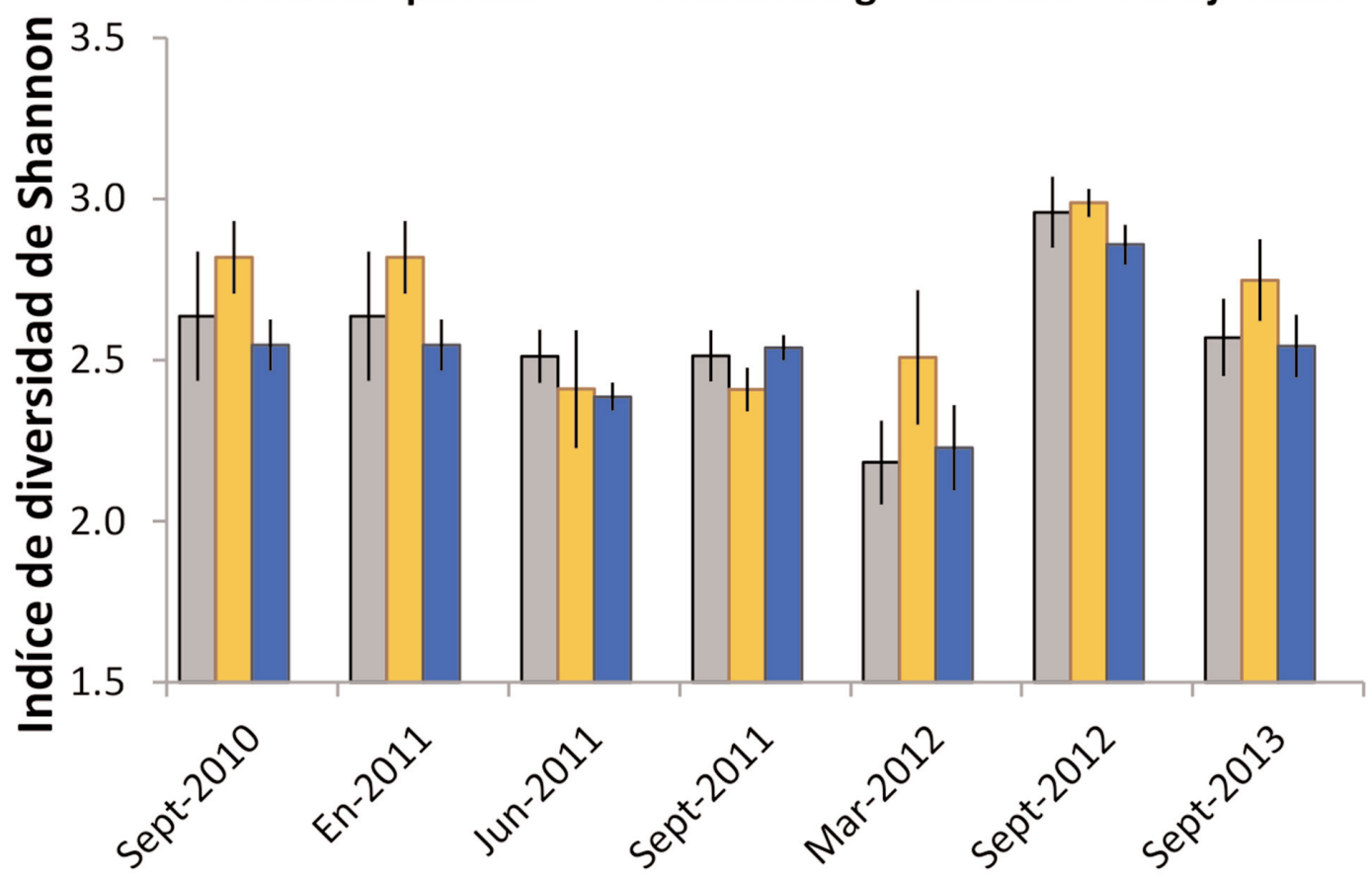



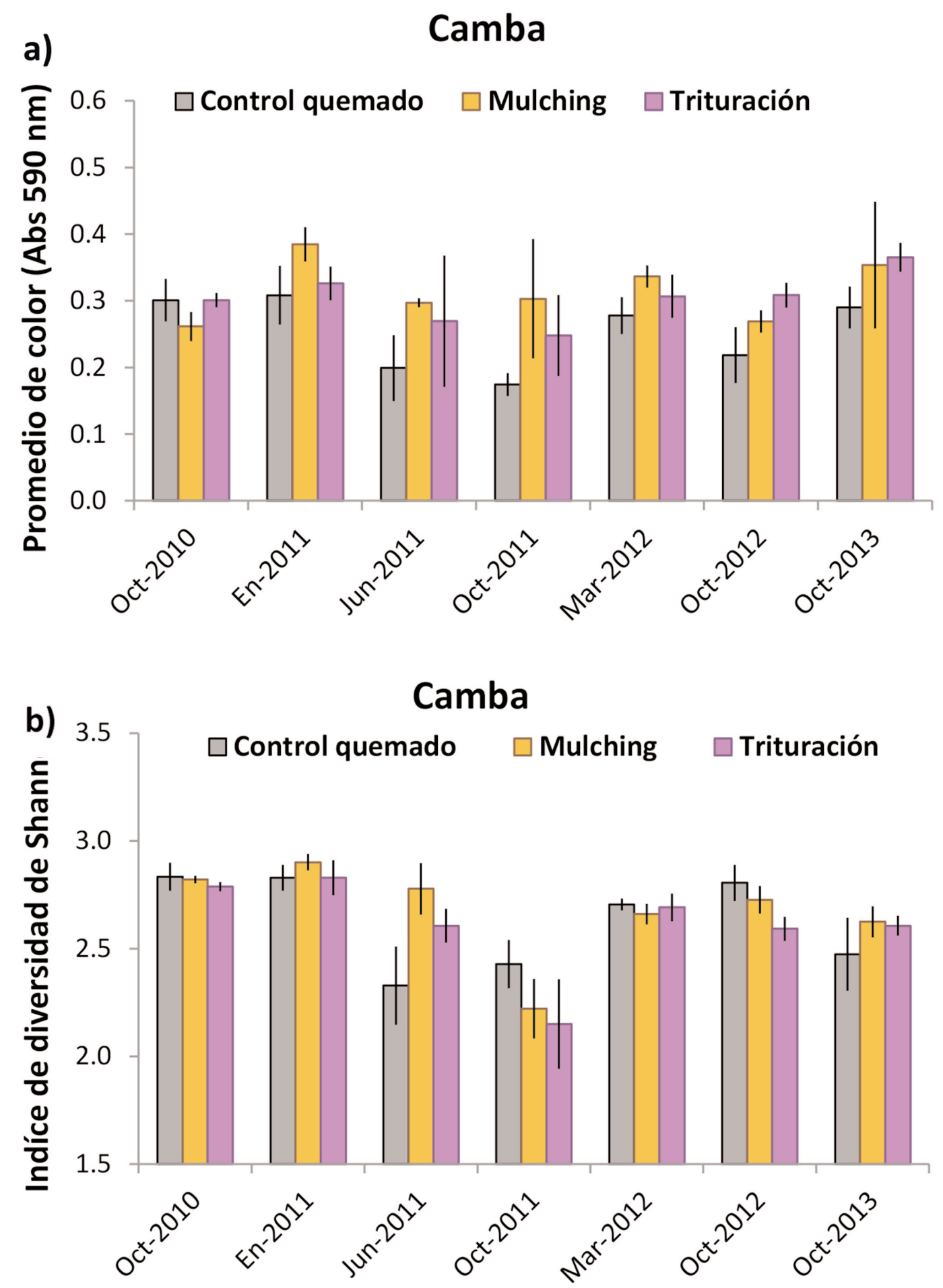

Figura 3. Efectos de los tratamientos de rehabilitación post-fuego aplicados en los dispositivos experimentales de Piñor, Boiro y Camba en: a) utilización microbiana de sustratos de $\mathrm{C}$ (promedio de color) y b) índice de diversidad de Shannon del suelo mineral superficial $(0-2 \mathrm{~cm})$ a lo largo de 3 años de estudio. Letras distintas en cada fecha indican diferencias significativas $(\mathrm{p}<0.05)$ según el test de Student-Newman-Keuls. 


\section{Agradecimientos}

Este trabajo ha sido financiado por el INIA a través del proyecto RTA201100065-C02-01. (Rehabilitación y restauración post-incendio: Efectos en el tiempo sobre la recuperación de la vegetación afectada y su inflamabilidad y en las características del suelo), cofinanciado con fondos FEDER. La selección de las áreas de estudio, instalación de parcelas y mediciones de campo fue realizada con el apoyo de Antonio Arellano al que siempre agradeceremos su esfuerzo y dedicación. También nuestro agradecimiento a todos los que han colaborado en los trabajos de campo y laboratorio, especialmente, Dolores Cernadas, Bárbara García, Elena Pérez, José R. González y Jesús Pardo.

\section{Referencias bibliográficas}

Badía, D., Marti, C., 2000. Seeding and mulching treatments as conservation measures of two burned soils in the central Ebro valley, NE Spain. Arid Soil Res. Rehabil. 13, 219-232.

Barreiro, A., Fontúrbel, M.T., Lombao, A., Martín, A., Vega, J.A., Fernández, C., Carballas, T., Díaz-Raviña, M., 2015. Using phospholipid fatty acid and community level physiological profiling techniques to characterize soil microbial communities following an experimental fire and different stabilization treatments. Catena 135, 419-429.

Bautista, S., Bellot, J. Vallejo, V.R., 1996. Mulching treatment for postfire soil conservation in a semiarid ecosystem. Arid Soil Res. Rehabil. 10, 235-242.

Bautista, S., Robichaud, P.R., Bladé, C., 2009. Post-fire mulching. In: Cerdá, A., Robichaud, P. (eds), Fire effects on soils and restoration strategies. Science Publishers, pp. 353-372.

Benito, E., Varela, M.E., Rodríguez-Alleres, M., 2014. Wildfire effects on soil erodibility in Galicia. Cuadernos de Investigación Geográfica 40, 353-370.

Busse, M.D., Sanchez, F.G., Ratcliff, A.W., Butnor, J.R., Carter, E.A., 2009. Soil carbon sequestration and changes in fungal and bacterial biomass following incorporation of forest residues. Soil Biol. Biochem. 41, 220-227.

Certini, G., 2005. Effects of fire on properties of forest soils, a review. Oecologia, 143, 1-10.

D’Ascoli, R., Rutigliano, F.A., De Pascale, R.A., Gentile, A., Virzo De Santo, A., 2005. Functional diversity of the microbial community in Mediterranean maquis soils as affected by fires. Int. J. Wildl. Fire, 14, 355-363.

Díaz-Raviña, M., Martín, A., Barreiro, A., Lombao, A., Iglesias, L., Díaz-Fierros, F., Carballas, T., 2012. Mulching and seeding treatments for post-fire soil stabilisation in NW Spain: short-term effects and effectiveness. Geoderma, 191, 31-39.

Fernández, C., Vega, J.A., Jiménez, E., Fonturbel, M.T., 2011. Effectiveness of three post-fire treatments at reducing soil erosion in Galicia (NW Spain). Int JWildland Fire, 20, 104-114.

Fernández, C., Vega, J.A., Jiménez, E., Vieira, D.C.S., Merino, A., Ferreiro, A., Fontúrbel, T., 2012. Seeding and mulching + seeding effects on post-fire runoff, soil erosion and species diversity in Galicia (NW Spain). Land Degrad. Dev. 23, 150-156.

Fernández, C.; Vega, J.A., 2013. Impacto de dos tipos de mulch para el control de la erosión en la recuperación de la cubierta vegetal en un área incendiada del NW España. $6^{\circ}$ Congreso Forestal Español. 
Fernández, C.; Vega, J.A., 2015. Modelling the effect of soil burn severity on soil erosion at hillslope scale in the first year following wildfire in NW Spain. Earth Surf. Process. Landforms DOI: 10.1002/esp.3876

Fontúrbel, M.T., Barreiro, A., Vega J.A., Martín, A., Jiménez, E., Carballas, T., Fernández, C., Díaz-Raviña, M., 2012. Effects of an experimental fire and post-fire stabilization treatments on soil microbial communities. Geoderma, 191, 51-60.

García, E., 2013. Estrategias para la recuperación de suelos degradados en ambientes semiáridos: adición de dosis elevadas de residuos orgánicos de origen urbano y su implicación en la fijación de carbono. Tesis doctoral. Universidad de Murcia.

Garland, J.L., 1996. Analytical approaches to the characterization of samples of microbial communities using patterns of potential carbon source utilization. Soil Biol. Biochem. 28, 223-230.

Gómez-Rey, M.X., Couto-Vázquez, A., García-Marco, S., González-Prieto, S.J., 2013a. Impact of fire and post-fire management techniques on soil chemical properties. Geoderma 195-196:155-164.

Gómez-Rey, M.X., Couto-Vázquez, A., García-Marco, S., Vega, J.A., González-Prieto, S.J., 2013b. Reduction of nutrient losses with eroded sediments by post-fire soil stabilization techniques. Int. J. Wildland Fire, 22, 696-706.

González Pérez, J.A., González Vila, J.F., Almendros. G., Knicker, H., 2004.The effect of fire on soil organic matter- a review. Environ. Int. 30, 855-870.

Huang, Z.Q., Xu, Z.H., Chen, C.R., 2008. Effect of mulching on labile soil organic matter pools, microbial community functional diversity and nitrogen transformations in two hardwood plantations of subtropical Australia. Appl. Soil Ecol. 40, 229-239.

Lombao, A., Díaz-Raviña, M., Martín, A., Barreiro, A., Fontúrbel, M.T., Vega, J.A., Fernández, C., Carballas, T., 2015. Influence of straw application on the properties of a soil affected by a forest wildfire. Spanish J. Soil Sci. 5, 26-40.

Martín, A., Díaz-Raviña, M., Carballas, T., Vega, J.A., Jiménez, E., Fonturbel, T., 2010. Caracterización físico-química de suelo quemado con diferentes tratamientos de rehabilitación. En: Actas Jornadas Internacionales sobre Investigación y Gestión para la protección del suelo y restauración de los ecosistemas forestales afectados por incendios forestales. FUEGORED 2010.

Mataix-Solera, J., Guerrero, C., García-Orenes, F., Bárcenas, G.M., Torres, M.P., 2009. Forest fire effects on soil microbiology. In: Cerdà, A., Robichaud, P.R. (eds.), Fire Effects on Soils and Restoration Strategies. Land Reconstruction and Management Series, vol. 5. Science Publishers, Enfield, Jersey, Plymouth, pp. 133-175.

Pietikäinen, J., Hiukka, R., Fritze, H., 2000. Does short-term heating of forest humus changes its properties as a substrate for microbes? Soil Biol. Biochem. 32, 277-288.

Pinaya, I., Soto, B., Arias, M., Díaz-Fierros, F., 2000. Revegetation of burnt areas: Relative effectiveness of native and commercial seed mixtures. Land Degrad Dev. 11, 93-98.

Robichaud, P.R., Lewis, S.A., Wagenbrenner, J.W., Ashmun, L.E., Brown, R.E., 2013. Postfire mulching for runoff and erosion mitigation. Part I: Effectiveness at reducing hillslope erosion rates. Catena 105, 75-92.

San Miguel, J., Camia, A., 2009. Forest fires at a glance: facts, figures and trends in the EU. In: Birot, Y. (ed.), Living with wildfires: what science can tell us - A contribution to the Science-Policy Dialogue. European Forest Institute, Discussion Paper 15, 11-18.

Staddon, W.J., Duchesne, L.C., Trevors, J.T., 1997. Microbial diversity and community struc- 
ture of post-disturbance forest soils as determined by sole-carbon-source-utilization patterns. Microb. Ecol. 34, 125-130.

Trasar-Cepeda, M.C., Gil, F., Leirós de la Peña, C., 2003. Determinación de la actividad fosfatasa del suelo. En: García, C., Gil. F., Hernández, T., Trasar, M.C. (eds.), Técnicas de Análisis de Parámetros Bioquímicos en Suelos: Medidas de Actividades Enzimáticas y Biomasa Microbiana. Mundi-Prensa, Madrid. pp. 55 - 76.

Vance, E.D., Brookes, P.C. y Jenkinson, D.S., 1987. A extraction method for measuring soil microbial biomass C. Soil Biol. Biochem. 19, 703-707.

Vega, J.A., Fontúrbel, T., Fernández, C., Arellano, A., Díaz-Raviña, M., Carballas, T., Martín, A., González-Prieto, S., Merino, A., Benito, E., 2013 a. Acciones urgentes contra la erosión en áreas forestales quemadas. Guía para su planificación en Galicia. Santiago de Compostela. Andavira.

Vega, J.A., Fontúrbel, M.T., Merino, A., Fernández, C., Ferreiro, A., Jiménez, E., 2013 b. Testing the ability of visual indicators of soil burn severity to reflect changes in soil chemical and microbial properties in pine forests and shrubland. Plant Soil 369, 73-91.

Vega, J.A., Fernández, C., Fonturbel, T., González-Prieto, S., Jiménez, E., 2014. Testing the effects of straw mulching and herb seeding on soil erosion after fire in a gorse shrubland, Geoderma 223-225, 79-87.

Vega, J.A., Fernández, C., Fonturbel, T., 2015. Comparing the effectiveness of seeding and mulching + seeding in reducing soil erosion after a high severity fire in Galicia (NW Spain). Ecol. Eng. 74, 206-212. 